\title{
A study of the oxygen and carbon dioxide requirements of thermophilic campylobacters
}

\author{
FJ BOLTON, D COATES \\ From the Public Health Laboratory, Preston Infirmary, Meadow Street, Preston, Lancashire PR1 6PS
}

SUMMARY The oxygen and carbon dioxide requirements of different biotypes of thermophilic campylobacters were investigated by means of (a) quantitative studies, and (b) total growth studies. Oxygen tolerance of the five test organisms differed markedly and varied with the carbon dioxide concentration. At most carbon dioxide concentrations tested, Campylobacter jejuni strains NCTC 11168 and NCTC 11392 tolerated 21\% oxygen (growth reduced), C coli NCTC 11353 tolerated 15\% oxygen (growth reduced), and $C$ jejuni ATCC 3036 and (nalidixic acid resistant thermophilic campylobacter) NCTC 11352 tolerated 10\% oxygen (growth not reduced). Total growth studies indicated that $10 \%$ oxygen was the optimal concentration for growth of the five test organisms. All exhibited a requirement for carbon dioxide, and only $C$ jejuni strains NCTC 11168 and NCTC 11392 tolerated its absence (growth reduced), when the oxygen concentration was low. The studies indicated that atmospheres containing $5 \%$ to $10 \%$ oxygen and $1.0 \%$ to $10 \%$ carbon dioxide are suitable for growth of the various biotypes of thermophilic campylobacters.

The oxygen and carbon dioxide concentrations produced in anaerobic jars by variations of the evacuation-replacement technique were determined and suitable practices identified.

Although it is known that campylobacters are microaerophilic, little information is available on the optimum and range of oxygen and carbon dioxide concentrations required for culture of the different biotypes. Kiggens and Plastridge ${ }^{1}$ reported that an atmosphere of $5 \%$ oxygen and $10 \%$ carbon dioxide in nitrogen was optimal for Campylobacter fetus (Vibrio fetus of bovine origin), and Dekeyser et al ${ }^{2}$ and Skirrow ${ }^{3}$ found that similar mixtures were suitable for $C$ jejuni and $C$ coli.

Several methods of producing a microaerobic atmosphere suitable for campylobacters have been examined. Butzler \& Skirrow ${ }^{4}$ drew a partial vacuum $(500 \mathrm{~mm} \mathrm{Hg})$ in a carbon dioxide incubator or anaerobic jar (without catalyst) and refilled with a $15 \%$ carbon dioxide $/ 85 \%$ nitrogen or hydrogen mixture (evacuation-replacement technique). Other workers have evacuated to different negative pressures and refilled with different gas mixtures. DeBoeck $^{5}$ incorporated the aerotolerant supplement (a mixture of ferrous sulphate, sodium matabisulphite and sodium pyruvate) of George $e t$ $a l^{6}$ into Butzler's medium ${ }^{4}$ and cultured specimens in a candle jar. A more convenient method is to use one of the commercial gas generating envelopes recently introduced. In a previous study ${ }^{7}$ we analysed the oxygen and carbon dioxide concentrations produced by these methods and tested the suitability of the atmospheres for culture of the different Campylobacter biotypes. ${ }^{8}$ The present study was carried out to determine the optimum and range of oxygen and carbon dioxide concentrations required for culture of the different biotypes.

\section{Material and methods}

\section{TEST ORGANISMS AND MEDIA}

The test organisms used were Campylobacter jejuni biotype 1 (8) NCTC 11168 and ATCC 3036, C jejuni biotype 2 (8) NCTC 11392, $C$ coli NCTC 11353, and nalidixic acid resistant thermophilic campylobacter (NARTC) NCTC 11352.

The organisms were grown on Columbia agar (Oxoid CM331) containing 5\% horse blood for $24 \mathrm{~h}$ at $42^{\circ} \mathrm{C}$ in a microaerobic atmosphere produced in an anaerobic jar (catalyst removed) by evacuation to $500 \mathrm{~mm} \mathrm{Hg}$ and refilling with $10 \%$ carbon dioxide in nitrogen. Bacteria were harvested into $0.1 \%$ peptone water (Oxoid L37) and the suspensions standardised to approximately $2 \times 10^{8}$ colony forming 
units (CFU) per ml with a Perkin-Elmer model $6 / 20$ spectrophotometer at a wavelength of $450 \mathrm{~nm}$. Tenfold dilutions over six steps were made in $0.1 \%$ peptone water.

The recovery medium used in tests was nutrient broth No 2 (Oxoid CM67) containing 2\% New Zealand agar and $5 \%$ lysed horse blood, prepared as plates or slopes in bijoux bottles. Plates were dried at room temperature.

\section{JARS}

Anaerobic jars (Baird \& Tatlock) were used without catalysts. An adjustable needle valve was fitted to each lid and side arm for input of gas mixtures and removal of gas samples respectively.

\section{PRODUCTION OF DIFFERENT MICROAEROBIC ATMOSPHERES}

Gas mixtures containing $<0.5 \%$ to $21 \%$ oxygen and 0 to $15 \%$ carbon dioxide were made up in 201 plastic bags from oxygen, carbon dioxide and nitrogen in cylinders (BOC special gases). After gas analysis the mixtures were introduced into jars that had been evacuated to $650 \mathrm{~mm} \mathrm{Hg}$ and which contained cultures of test organisms. A gas sample from each jar was taken for analysis and where necessary a second evacuation-replacement cycle was carried out.

\section{ANALYSIS OF GAS SAMPLES}

Gas samples were removed from the jars using a vacuum pump which was connected directly to the analysers. Carbon dioxide was determined with a Lira Infrared Analyser, model 303 (Mine Safety Appliances Company, Pittsburgh, Pennsylvania, USA) set at a range of 0 to $10 \%$ and accurate to $0 \cdot 1 \%$. Concentrations $>10 \%$ were determined with Draeger gas detection equipment. Oxygen was determined with an oxygen analyser, model OA272 (Taylor Servomax, Sybron Corporation, Rochester, New York, USA) set at a range of 0 to $25 \%$ and accurate to $0.25 \%$.

\section{GROWTH OF TEST ORGANISMS IN DIFFERENT MICROAEROBIC ATMOSPHERES \\ Quantitative studies}

The ability of the various atmsopheres to support the growth of small numbers of test organisms was studied quantitatively using the method of Miles $e t$ al..$^{9}$ Plates of recovery medium were inoculated with the $10^{-5}$ and $10^{-6}$ dilutions of standardised suspensions using a 50-dropper pipette and incubated in the test jars for $48 \mathrm{~h}$ at $42^{\circ} \mathrm{C}$. A control set of plates was incubated in a jar evacuated to $500 \mathrm{~mm} \mathrm{Hg}$ and refilled with $10 \%$ carbon dioxide in nitrogen (routine laboratory procedure). Counts were made and a growth index calculated by dividing the counts obtained on the test plates by those on the control plates.

\section{Total growth studies}

The total growth of test organisms produced in the various atmospheres was determined by measuring the optical density of harvested suspensions. Two $\vec{\nabla}$ slopes of recovery medium were inoculated using a $\cong$ $1 / 300 \mathrm{ml}$ loop with the $10^{-3}$ dilution of a standar- के dised suspension and incubated with caps loosened $\vec{O}$ in the test jars for $48 \mathrm{~h}$ at $42^{\circ} \mathrm{C}$. After incubation $1 \mathrm{ml}$ of $0.5 \%$ formalin in $0.1 \%$ peptone water was $\vec{\omega}$ pipetted onto each slope and also onto an uninoculated slope (for blanking the spectrophotometer). ? Growth from each of the inoculated slopes was har- $\omega$ vested and $0.2 \mathrm{ml}$ of each suspension pipetted into $-v$ the well of a Linbro, flat-bottomed microtitre plate. 옹 The optical density of each suspension, relative to the blank, was measured using a microelisa mini $\frac{}{-}$ reader model MR90 (Dynatech Instruments Ltd.) at $\vec{c}$ a wavelength of $490 \mathrm{~nm}$, and the mean calculated.

INVESTIGATION OF ATMOSPHERES PRODUCED BY DIFFERENT EVACUATION-REPLACEMENT TECHNIQUES

Jars were evacuated to $400,500,600$ and $700 \mathrm{~mm} \mathrm{Hg}$ and refilled with mixtures of $5 \%, 10 \%$, $15 \%$ and $20 \%$ carbon dioxide in nitrogen. Gas samples were then taken for analysis. All experiments $\stackrel{\square}{\mathbb{Q}}$ were done in duplicate.

\section{Results}

\section{QUANTITATIVE STUDIES}

The Table gives the growth indices obtained for the? test organisms in atmospheres of varying oxygen and carbon dioxide concentrations. Differences between the mean counts obtained in the test and control $O$ atmospheres were analysed by a two sample $t$ test

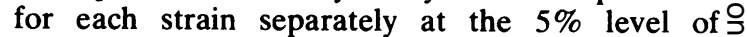
significance. Growth indices significantly different $D$ from 1.00 are asterisked in the Table.

In the absence of carbon dioxide $C$ 3036, $C$ coli and the NARTC strain all fol of grow on the test plates; $C$ jejuni strains NCTC 11168 and NCTC 11392 grew in atmospheres con- $\tilde{\omega}$ taining $1 \%$ and $5 \%$ oxygen but the growth indices were significantly less than $1 \cdot 00$.

In atmospheres containing less than $0.5 \%$ oxygen $C$ jejuni NCTC 11168 and ATCC 3036 grew satis- $\stackrel{?}{+}$ factorily in carbon dioxide concentrations ranging ${ }^{\circ}$ from $2.5 \%$ to $15 \%$, and the other test organisms in carbon dioxide concentrations of $1 \%$ to $15 \%$. In atmospheres containing $1 \%$ oxygen, all of the test $\stackrel{\mathbb{Q}}{\Omega}$ organisms grew satisfactorily in carbon dioxide con- $\overline{-}$ centrations ranging from $1 \%$ to $15 \%$. In atmos- 
Growth indices of test organisms (counts obtained in test atmospheres/counts obtained in the control atmosphere) cultured in atmospheres of varying oxygen and carbon dioxide concentrations.

\begin{tabular}{|c|c|c|c|c|c|c|c|}
\hline \multirow[t]{2}{*}{ Test organism } & \multirow[t]{2}{*}{ \% oxygen } & \multicolumn{6}{|c|}{ \% carbon dioxide } \\
\hline & & 0 & 1 & $2 \cdot 5$ & 5 & 10 & 15 \\
\hline $\begin{array}{l}C \text { jejuni } \\
\text { NCTC } 11168\end{array}$ & $\begin{array}{c}<0 \cdot 5 \\
1 \\
5 \\
10 \\
15 \\
21\end{array}$ & $\begin{array}{l}\text { Not done } \\
0 \cdot 07^{*} \\
0 \cdot 06^{*} \\
0 \\
0 \\
0\end{array}$ & $\begin{array}{l}0.53^{*} \\
1.21 \\
0.99 \\
1.13 \\
0.35^{*} \\
0.34^{*}\end{array}$ & $\begin{array}{l}0.89 \\
1.18 \\
0.98 \\
1.15 \\
0.58^{*} \\
0.41^{*}\end{array}$ & $\begin{array}{l}1.03 \\
1 \cdot 16 \\
0.99 \\
1.12 \\
0 \cdot 60^{*} \\
0 \cdot 39^{*}\end{array}$ & $\begin{array}{l}0.84 \\
1.17 \\
0.98 \\
1.10 \\
0.71^{*} \\
0.30^{*}\end{array}$ & $\begin{array}{l}1 \cdot 10 \\
1.16 \\
0.98 \\
1.15 \\
0.76 \\
0 \cdot 28^{*}\end{array}$ \\
\hline $\begin{array}{l}\text { C jejuni } \\
\text { ATCC } 3036\end{array}$ & $\begin{array}{c}<0 \cdot 5 \\
1 \\
5 \\
10 \\
15 \\
21\end{array}$ & $\begin{array}{l}\text { Not done } \\
0 \\
0 \\
0 \\
0 \\
0\end{array}$ & $\begin{array}{l}0.53^{*} \\
1.02 \\
0.96 \\
0.97 \\
0 \\
0\end{array}$ & $\begin{array}{l}0.98 \\
1.02 \\
0.81 \\
0.95 \\
0 \\
0\end{array}$ & $\begin{array}{l}1.15 \\
1.01 \\
0.78 \\
0.79 \\
0 \\
0\end{array}$ & $\begin{array}{l}1.02 \\
0.98 \\
0.96 \\
0.78 \\
0.49^{*} \\
0\end{array}$ & $\begin{array}{l}0.93 \\
0.97 \\
0.63^{*} \\
0.51^{*} \\
0 \\
0\end{array}$ \\
\hline $\begin{array}{l}\text { C jejuni } \\
\text { NCTC } 11392\end{array}$ & $\begin{array}{c}<0 \cdot 5 \\
1 \\
5 \\
10 \\
15 \\
21\end{array}$ & $\begin{array}{l}\text { Not done } \\
0 \cdot 61^{*} \\
0 \cdot 75^{*} \\
0 \\
0 \\
0\end{array}$ & $\begin{array}{l}1.02 \\
0.92 \\
0.95 \\
1.03 \\
0.81 \\
0.57^{*}\end{array}$ & $\begin{array}{l}0.96 \\
0.91 \\
1.01 \\
0.98 \\
0.82 \\
0.50^{*}\end{array}$ & $\begin{array}{l}1.17 \\
0.92 \\
1.05 \\
1.15 \\
0.68^{*} \\
0.48^{*}\end{array}$ & $\begin{array}{l}1 \cdot 16 \\
0.89 \\
1.03 \\
1.11 \\
0.72^{*} \\
0.38^{*}\end{array}$ & $\begin{array}{l}1.02 \\
0.85 \\
0.88 \\
0.86 \\
0.44^{*} \\
0\end{array}$ \\
\hline $\begin{array}{l}C \text { coli } \\
\text { NCTC } 11353\end{array}$ & $\begin{array}{c}<0 \cdot 5 \\
1 \\
5 \\
10 \\
15 \\
21\end{array}$ & $\begin{array}{l}\text { Not done } \\
0 \\
0 \\
0 \\
0 \\
0\end{array}$ & $\begin{array}{l}1.02 \\
0.98 \\
0.78 \\
1.02 \\
0 \\
0\end{array}$ & $\begin{array}{l}1.09 \\
0.96 \\
0.79 \\
0.85 \\
0.89 \\
0\end{array}$ & $\begin{array}{l}1.09 \\
0.96 \\
0.97 \\
0.95 \\
0.29^{*} \\
0\end{array}$ & $\begin{array}{l}1.09 \\
0.92 \\
1.01 \\
0.86 \\
0.32^{*} \\
0.08^{*}\end{array}$ & $\begin{array}{l}1.05 \\
0.92 \\
0.90 \\
0.88 \\
0.12^{*} \\
0\end{array}$ \\
\hline $\begin{array}{l}\text { NARTC } \\
\text { NCTC } 11352\end{array}$ & $\begin{array}{c}<0 \cdot 5 \\
1 \\
5 \\
10 \\
15 \\
21\end{array}$ & $\begin{array}{l}\text { Not done } \\
0 \\
0 \\
0 \\
0 \\
0\end{array}$ & $\begin{array}{l}0.99 \\
1.02 \\
1.01 \\
1.03 \\
0 \\
0\end{array}$ & $\begin{array}{l}1 \cdot 00 \\
0 \cdot 97 \\
1 \cdot 10 \\
0 \cdot 91 \\
0 \\
0\end{array}$ & $\begin{array}{l}1 \cdot 01 \\
0.82 \\
1.06 \\
0 \cdot 86 \\
0 \\
0\end{array}$ & $\begin{array}{l}0.88 \\
0.77 \\
1.35 \dagger \\
0.95 \\
0 \\
0\end{array}$ & $\begin{array}{l}1 \cdot 02 \\
0 \cdot 79 \\
1 \cdot 10 \\
0 \cdot 88 \\
0 \\
0\end{array}$ \\
\hline
\end{tabular}

* Growth index significantly less than 1.00 (5\% level).

tGrowth index significantly greater than 1.00 (5\% level).

0: No growth of test organism on test plate.

pheres containing $5 \%$ to $10 \%$ oxygen. $C$ jejuni strains NCTC 11168 and NCTC 11392, C coli and the NARTC strain all grew satisfactorily in carbon dioxide concentrations ranging from $1 \%$ to $15 \%$, and $C$ jejuni ATCC 3036 in carbon dioxide concentrations of $1 \%$ to $10 \%$. The growth index of the NARTC strain was significantly greater than 1.00 when grown in an atmosphere containing $5 \%$ oxygen and $10 \%$ carbon dioxide. In atmospheres containing $15 \%$ oxygen growth on the test plates was generally much reduced or absent; $C$ jejuni NCTC 11168 grew satisfactorily in $15 \%$ carbon dioxide, $C$ jejuni NCTC 11392 in $1 \%$ and $2.5 \%$ carbon dioxide, and the $C$ coli strains in $2.5 \%$ carbon dioxide. In atmospheres containing $21 \%$ oxygen in all cases growth on the test plates was either very much reduced or absent.

\section{TOTAL GROWTH STUDIES}

Figure 1 shows the total growth patterns of the five test organisms on slopes of recovery medium after incubation in atmospheres containing oxygen con- centrations ranging from $1 \%$ to $21 \%$, and carbon dioxide concentrations ranging from $0 \%$ to $15 \%$.

Figure 1a demonstrates that in the absence of carbon dioxide $C$ jejuni NCTC 11168 grew poorly or not at all, depending on the oxygen concentration. However, in the presence of $1 \%$ to $15 \%$ carbon dioxide the total growth was good and tended to increase slightly with carbon dioxide concentration. With regard to oxygen concentration, the total growth was good over the range $1 \%$ to $21 \%$ with a peak at $10 \%$. An atmosphere containing $5 \%$ to $15 \%$ carbon dioxide and $10 \%$ oxygen was optimal.

Figure $1 \mathrm{~b}$ demonstrates that the total growth patterns of $C$ jejuni ATCC 3036 in different concentrations of carbon dioxide were variable. The organism failed to grow in the absence of carbon dioxide, and only grew in the presence of $15 \%$ oxygen when the carbon dioxide concentration was $10 \%$. An atmosphere containing $1 \%$ to $5 \%$ carbon dioxide and $10 \%$ oxygen was optimal.

Figure $1 \mathrm{c}$ demonstrates that the total growth patterns of $C$ jejuni NCTC 11392 were similar to those 

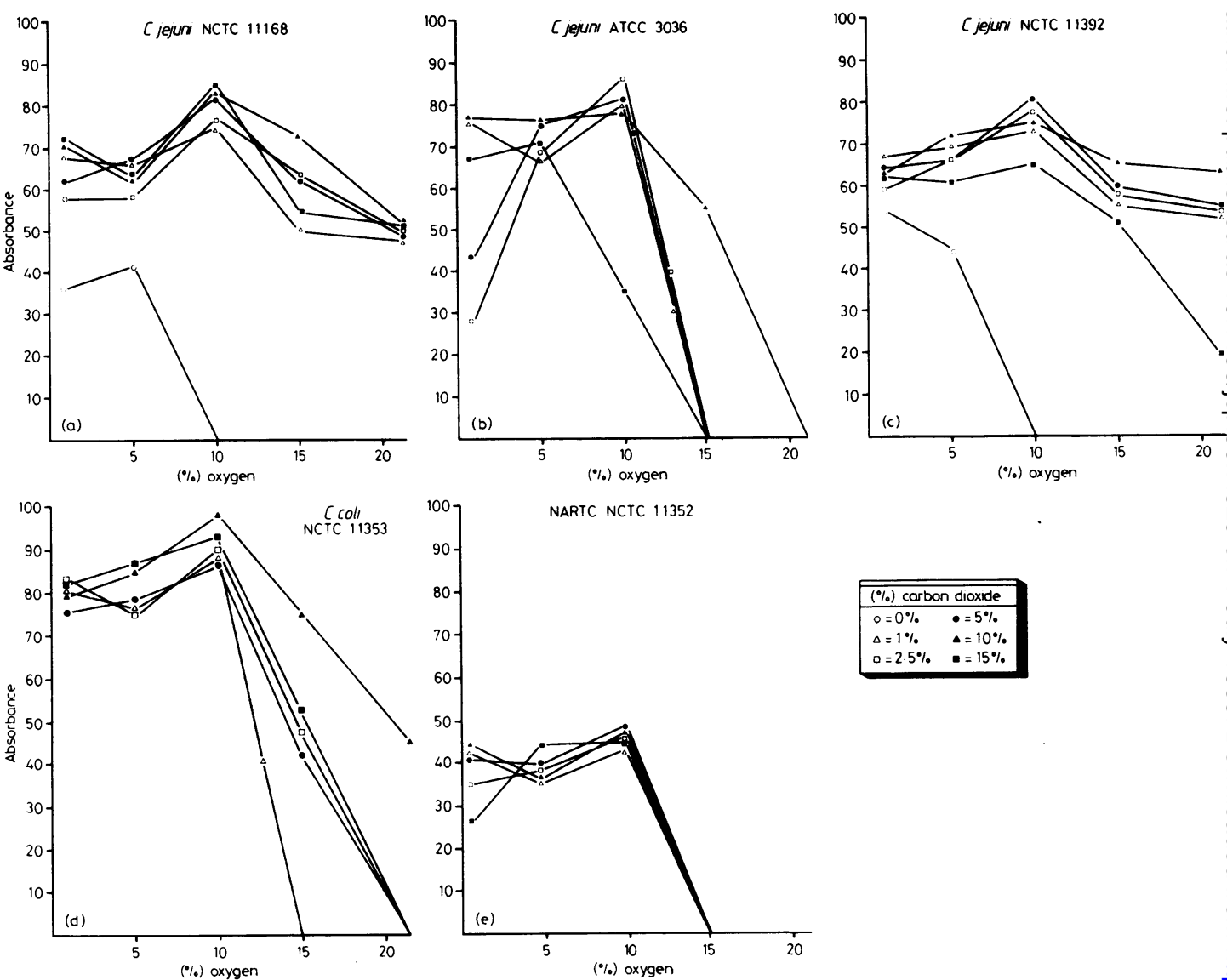

Fig. 1 Total growth patterns of test organisms on slopes after incubation in atmospheres of varying oxygen and carbon dioxide concentrations.

of $C$ jejuni NCTC 11168; an atmosphere containing $2.5 \%$ to $10 \%$ carbon dioxide and $10 \%$ oxygen was optimal.

Figure 1d demonstrates that the total growth patterns of $C$ coli NCTC 11353 in atmospheres containing $1 \%$ to $15 \%$ carbon dioxide were similar over the oxygen range $1 \%$ to $10 \%$ but diverged at higher oxygen concentrations. The organism failed to grow in the absence of carbon dioxide, and only grew in the presence of $21 \%$ oxygen when the carbon dioxide concentration was $10 \%$. An atmosphere containing $2.5 \%$ to $15 \%$ carbon dioxide and $10 \%$ oxygen was optimal.

Figure 1e demonstrates that the total growth patterns of NARTC NCTC 11352 in atmospheres containing $1 \%$ to $15 \%$ carbon dioxide were similar over the oxygen range $1 \%$ to $10 \%$. The organism failed to grow in the absence of carbon dioxide or in the presence of $15 \%$ oxygen. An atmosphere containing
$2.5 \%$ to $10 \%$ carbon dioxide and $10 \%$ oxygen was optimal.

\section{ATMOSPHERES PRODUCED BY DIFFERENT}

EVACUATION-REPLACEMENT TECHNIOUES

Figure 2 shows the oxygen and carbon dioxide con $N$ centrations produced in anaerobic jars evacuated to different pressures and then refilled with mixtures of $5 \%, 10 \%, 15 \%$ and $20 \%$ carbon dioxide in nitrogen. It can be seen that the oxygen concentration produced in the jars decreased as the evacuation pressure increased. The oxygen concentrations prots duced with different evacuation pressures were approximately $14 \%$ with $400 \mathrm{~mm} \mathrm{Hg}, 9.5 \%$ with $500 \mathrm{~mm} \mathrm{Hg}, 5 \%$ with $600 \mathrm{~mm} \mathrm{Hg}$ and $1.5 \%$ with $700 \mathrm{~mm} \mathrm{Hg}$. Conversely, the carbon dioxide con $\frac{?}{\Phi}$ centrations produced increased as the evacuation pressure increased, and also with the concentration 


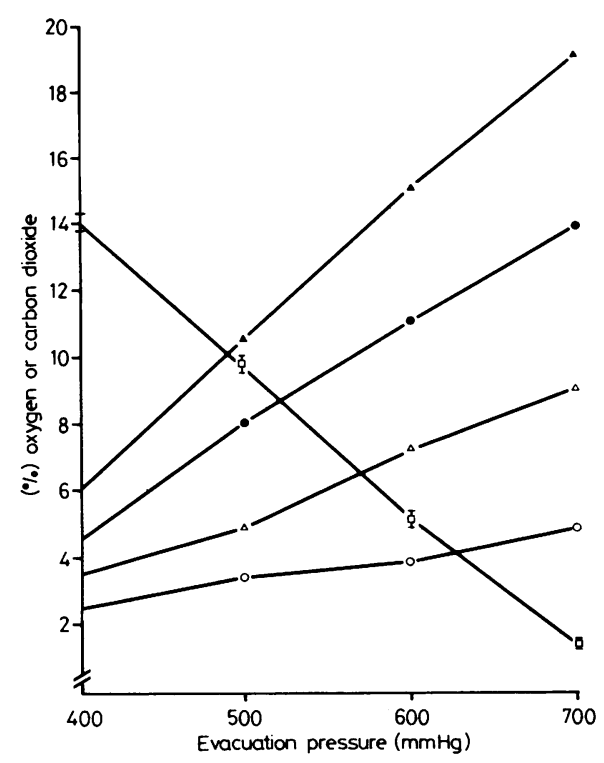

Fig. 2 Oxygen and carbon dioxide concentrations produced in anaerobic jars by different

evacuation-replacement techniques. $\phi_{1}=$ oxygen concentration range after replacement with 5-20\% carbon dioxide in nitrogen mixtures; $\mathrm{O}=$ carbon dioxide concentration after replacement with $5 \%$ carbon dioxide in nitrogen; $\Delta=$ carbon dioxide concentration after replacement with $10 \%$ carbon dioxide in nitrogen; $=$ carbon dioxide concentration after replacement with $15 \%$ carbon dioxide in nitrogen; $\mathbf{\Delta}=$ carbon dioxide concentration after replacement with $20 \%$ carbon dioxide in nitrogen.

of carbon dioxide present in the mixture used to refill the jar. After evacuation to $500 \mathrm{~mm} \mathrm{Hg}$, which is usually done, ${ }^{4}$ the carbon dioxide concentrations produced after refilling with different mixtures were approximately $3.25 \%$ with $5 \%$ carbon dioxide in nitrogen, $4.75 \%$ with $10 \%$ carbon dioxide in nitrogen, $8 \%$ with $15 \%$ carbon dioxide in nitrogen, and $10.5 \%$ with $20 \%$ carbon dioxide in nitrogen.

\section{Discussion}

The thermophilic campylobacters are microaerophilic, and many different methods of producing microaerobic atmospheres have been proposed for their isolation. In a previous study ${ }^{7}$ we found that the oxygen and carbon dioxide concentrations present in atmospheres produced by the different methods varied markedly. Hence, in order to ascertain which methods were best, we set out to determine the optimum and range of oxygen and carbon dioxide concentrations required for culture of the different biotypes of thermophilic campylobacters.

The present study has demonstrated that the mic- roaerophilic nature of different thermophilic campylobacters varies greatly, and that carbon dioxide is important for growth. $C$ jejuni strains NCTC 11168 (biotype 1) and NCTC 11392 (biotype 2) were the most aerotolerant test organisms which grew over the oxygen concentration range $<0.5 \%$ to $21 \%$ but with colony counts generally reduced in $\geqslant 15 \%$ oxygen. $C$ coli NCTC 11353 was the next most aerotolerant test organism which grew over the oxygen concentration range $<0.5 \%$ to $15 \%$ at most carbon dioxide concentrations tested but with colony counts generally reduced in $15 \%$ oxygen; slight growth occurred in an atmosphere of $21 \%$ oxygen and $10 \%$ carbon dioxide. $C$ jejuni ATCC 3036 (biotype 1) was one of the least aerotolerant test organisms which grew well over the oxygen concentration range $<0.5 \%$ to $10 \%$, but which generally failed to grow in $15 \%$ oxygen. NARTC NCTC 11352 was the least aerotolerant test organism which grew well over the oxygen concentration range $<0.5 \%$ to $10 \%$, but not at all in $15 \%$ oxygen. Total growth studies showed that atmospheres of $5 \%$ to $10 \%$ oxygen and $1 \%$ to $10 \%$ carbon dioxide produced very good growth of all of the test organisms.

In our previous study ${ }^{7}$ we measured the oxygen and carbon dioxide concentrations present in atmospheres produced by different methods of achieving microaerobiosis. An Oxoid BR60 envelope produced $8.5 \%$ oxygen and $4.8 \%$ carbon dioxide in an Oxoid 3.51 jar. A BBL CampyPak envelope produced $5.8 \%$ oxygen and $>10 \%$ carbon dioxide in a BBL 2.51 jar. The present studies show that both of these methods produce atmospheres satisfactory for growth of the different biotypes. The evacuation-replacement technique is widely used to achieve microaerobiosis. Butzler and Skirrow ${ }^{4}$ recommended an evacuation pressure of $500 \mathrm{~mm} \mathrm{Hg}$ and refilling with $15 \%$ carbon dioxide in nitrogen. Our assays have shown (Fig. 2) that this procedure will produce an atmosphere of approximately $9.75 \%$ oxygen and $8.0 \%$ carbon dioxide which is satisfactory for growth of the different biotypes. Our results show that evacuation pressures of $500-600 \mathrm{~mm} \mathrm{Hg}$ and refilling with mixtures of $5 \%$ to $15 \%$ carbon dioxide in nitrogen should produce atmospheres satisfactory for growth of the different biotypes. In our previous study ${ }^{7}$ a candle was found to produce $17.0 \%$ to $18.5 \%$ oxygen and $1.2 \%$ to $1.5 \%$ carbon dioxide concentrations in jars of different volume. In the present study it was found that $C$ jejuni strains NCTC 11168 and NCTC 11392 generally tolerated $21 \%$ oxygen, $C$ coli NCTC 11353 generally tolerated $15 \%$ oxygen, and $C$ jejuni ATCC 3036 and NARTC NCTC 11352 tolerated $10 \%$ oxygen. In previous studies with these organ- 
isms $^{710}$ only $C$ jejuni strains NCTC 11168 and NCTC 11392 grew in a candle jar, which conforms with the above findings and demonstrates that a candle jar is only suitable for the growth of relatively aerotolerant strains.

We wish to thank the staff of the Biological Science Department, Preston Polytechnic for their assistance and for the loan of the gas analysers used in this study.

\section{References}

' Kiggins EM, Plastridge WN. Effect of gaseous environment on growth and catalase content of Vibrio fetus cultures of bovine origin. J Bacteriol 1956;72:397-400.

${ }^{2}$ Dekeyser P, Gossuin-Detrain M, Butzler JP, Sternon J. Acute enteritis due to related vibrio: first positive stool cultures. $J$ Infect Dis 1972;125:390-2.

${ }^{3}$ Skirrow MB. Campylobacter enteritis: a "new" disease. $\mathrm{Br}$ Med J 1977;ii:9-11.
4 Butzler JP, Skirrow MB. Campylobacter enteritis. Clin Gastroenterol 1979;8:737-65.

${ }^{5}$ DeBoeck M. Simplified isolation techniques for Campylobacter jejuni. In: Newell DG, ed. Campylobacter: epidemiology, pathogenesis and biochemistry. Lancaster, England: MTP Press, 1982:71-2.

- George HA, Hoffman PS, Smibert RM, Krieg NR. Improved media for growth and aerotolerance of Campylobacter fetus. $J$ Clin Microbiol 1978;8:36-41.

' Bolton FJ, Coates D. A comparison of microaerobic systems for the culture of Campylobacter jejuni/coli. Eur J Clin Microbiol 1983;2:105-10.

Skirrow MB, Benjamin J. Differentiation of enteropathogenic Campylobacter. J Clin Pathol 1980;33:1122.

9 Miles AA, Misra SS, Irwin JO. The estimation of the bactericidal power of the blood. J Hyg (Camb) 1938;38:732-49.

${ }^{10}$ Bolton FJ, Coates D. Development of a blood-free Campylobacter medium: screening tests on basal media and supplements, and the ability of selected supplements to facilitate aerotolerance. J Appl Bacteriol 1983;54:115-25.

Requests for reprints to: Mr FJ Bolton, Public Health Laboratory, Preston Infirmary, Meadow Street, Preston, Lancashire PR1 6PS, England.

\section{Letters to the Editor}

\section{Pseudohyponatremia and hyperviscosity}

Serum from patients with hyperviscosity have low sodium values when analysed by flame photometry. ${ }^{1}$ The reason for the spuriously low sodium is related to problems with sample aspiration and dilution by decrease in plasma water due to high protein concentration. ${ }^{1-3}$ Methods that are available for measuring serum sodium include the traditional technique of flame photometry and more recently the use of an ion-selective electrode. When the serum sample is diluted before analysis by the electrode (indirect potentiometry), the serum sodium values would be expected to be low in the presence of hyperthe instrument and also because of the

proteinaemia and hyperviscosity. With direct potentiometry where no sample dilution takes place, no interference would be expected since the activity of sodium in the water phase only is being measured. The present study was undertaken to determine the magnitude of the decrease in serum sodium in viscous sera using flame photometric and indirect potentiometric measurements for sodium measurement.

\section{Patients and methods}

Samples were obtained from patients at the Vancouver General Hospital whose sera when analysed were noted to have increased viscosity at $37^{\circ} \mathrm{C}$ (Table). The range of serum viscosities was $3 \cdot 0-17 \cdot 8$

\section{Laboratory parameters in the patients with hyperviscosity}

\begin{tabular}{|c|c|c|c|c|c|}
\hline \multirow[t]{3}{*}{ Patient } & \multirow[t]{3}{*}{$\begin{array}{l}\text { Class and type } \\
\text { of paraprotein }\end{array}$} & \multirow[t]{3}{*}{$\begin{array}{l}\text { Serum viscosity } \\
\text { at } 37^{\circ} \mathrm{C}\end{array}$} & \multicolumn{3}{|c|}{$\begin{array}{l}\text { Serum sodium (normal } 135-145 \mathrm{mmol} / \mathrm{l} \text { ) } \\
\text { measured by }\end{array}$} \\
\hline & & & \multirow{2}{*}{$\begin{array}{l}\text { Flame } \\
\text { photometry }\end{array}$} & \multicolumn{2}{|c|}{ Potentiometry } \\
\hline & & & & Indirect & Direct \\
\hline $\begin{array}{r}1 \\
2 \\
3 \\
4 \\
5 \\
6 \\
7 \\
8 \\
9 \\
10 \\
11\end{array}$ & $\begin{array}{l}\text { IgG } \kappa \\
\operatorname{IgM} \kappa \\
\operatorname{IgM} \lambda \\
\operatorname{IgM} \kappa \\
\operatorname{IgG} \kappa \\
\operatorname{IgG} \kappa \\
\operatorname{IgG} \lambda \\
\operatorname{IgM} \lambda \\
\operatorname{IgM} \kappa \\
\operatorname{IgG} \boldsymbol{\lambda} \\
\operatorname{IgM} \kappa\end{array}$ & $\begin{array}{r}15 \\
4.8 \\
10.2 \\
3.3 \\
13.3 \\
17.8 \\
8.8 \\
11.1 \\
4.9 \\
5.7 \\
8.9\end{array}$ & $\begin{array}{l}130 \\
141 \\
143 \\
137 \\
128 \\
127 \\
132 \\
136 \\
133 \\
141 \\
136\end{array}$ & $\begin{array}{l}112 \\
141 \\
134 \\
138 \\
117 \\
101 \\
128 \\
128 \\
131 \\
137 \\
129\end{array}$ & $\begin{array}{l}142 \\
150 \\
150 \\
143 \\
144 \\
143 \\
146 \\
145 \\
144 \\
148 \\
146\end{array}$ \\
\hline
\end{tabular}

centistokes (normal is $<1 \cdot 8$ ). Serum sodium concentrations were measured in $\square$ duplicate by flame photometry (FP) (Beckman KLiNa flame: Beckman Instruments Inc, Fullerton, California, indirect potentiometry (IP) Beckman Astra, 음 Beckman Instruments Inc, Fullerton, $\varrho$ California) and by direct potentiometry $\overrightarrow{\vec{B}}$ (DP), (Nova-1 Na/K analyser, Nova Biomedical Inc, Newton, Massachusetts). $\vec{\supset}$ Serum viscosity relative to normal saline was measured using a Cannon Manning Viscometer. The differences in serum응 sodium obtained between measurements $\dot{\sigma}$ by DP and IP $\left(\triangle \mathrm{Na}_{1}\right)$ and DP and FP $\underline{3}$. $\left(\triangle \mathrm{Na}_{2}\right)$ were correlated with measurements of serum viscosity.

\section{Results}

Figure 1 shows the correlation $(r=0.95, p$ 을 $<0.001)$ between $\triangle \mathrm{Na}_{1}$ and serum viscos- $N$ ity. Figure 2 shows the correlation $(r=\Omega$ $0.71, \mathrm{p}<0.01$ ) between $\triangle \mathrm{Na}_{2}$ and serum $\mathrm{N}$ viscosity. For serum sodium measurements performed by indirect potentiometry, a $2 \omega$ $\mathrm{mmol} / \mathrm{l}$ decrease is seen for every one cen tistoke increase in serum viscosity.

\section{Discussion}

Dangerous pseudohyponatremia can occuro in patients with hyperviscosity (Table). We wish to draw attention to the fact that the $\frac{}{\mathbb{D}}$ degree of pseudohyponatremia is depen- $\varrho$ dent upon the technique that is used for 